\title{
From high anxiety trait to depression: a neurocognitive hypothesis
}

\author{
Carmen Sandi ${ }^{1}$ and Gal Richter-Levin ${ }^{2}$ \\ ${ }^{1}$ Laboratory of Behavioral Genetics, Brain Mind Institute, Ecole Polytechnique Federale de Lausanne (EPFL), Lausanne CH-1015, \\ Switzerland \\ ${ }^{2}$ The Institute for the Study of Affective Neuroscience, Brain and Behavior Laboratory, University of Haifa, Haifa 31905, Israel
}

\begin{abstract}
Although exposure to substantial stress has a major impact on the development of depression, there is considerable variability in the susceptibility of individuals to the adverse effects of stress. The personality trait of high anxiety has been identified as a vulnerability factor to develop depression. We propose here a new unifying model based on a series of neurocognitive mechanisms (and fed with crucial information provided by research on the fields of emotion, stress and cognition) whereby individuals presenting a high anxiety trait are particularly vulnerable to develop depression when facing stress and adversity. Our model highlights the importance of developing prevention programs addressed to restrain, in high anxious individuals, the triggering of a dysfunctional neurocognitive cascade while coping with stress.
\end{abstract}

\section{Introduction}

Individual differences in personality traits have been associated with both resilience to stress and stress-induced depression [1]. In particular, strong evidence highlights high anxiety trait as an important risk factor for the development (onset, severity and outcome) of depression $[2,3]$. However, it is not yet clear which are the mediating mechanisms, processes and factors whereby an individual with a highly anxious personality eventually evolves into clinical depression. To address this question it is important to also bear in mind that there is a considerable comorbidity between depression and anxiety disorders $(\sim 40-60 \%)$ [4] that seems to follow a temporal relationship, with anxiety disorders tending to precede the onset of depressive disorders and probably increasing the risk for subsequent depression [5].

Recent work suggests that the etiological factors (i.e. genetic and environmental) that influence high anxiety trait are the same as those that increase susceptibility for anxiety disorders and major depression (for review, see Ref. [3]). Two etiological hypotheses can account for such a picture: (i) that high anxiety trait, anxiety disorders and major depression are all directly caused by common etiological factors without necessary interrelations among themselves (Figure 1a); or (ii) that the etiological factors lead to high anxiety trait, which, in turn, predisposes to anxiety disorders and depression, contemplating the possibility that the first occurrence of anxiety disorders

Corresponding authors: Sandi, C. (carmen.sandi@epfl.ch); Richter-Levin, G. (galrichterlevin@gmail.com). facilitates the triggering of depression; this hypothesis also accounts for situations in which depression is directly developed from high anxiety trait (Figure 1b). Whereas the first hypothetical model proposes a direct link between etiological factors and the vulnerability to develop depression, the second model puts forward the personality trait of high anxiety as the vulnerability phenotype that mediates subsequent psychopathological developments. Given that substantial evidence indicates that high anxiety trait is a vulnerability factor to develop not only depression but also anxiety disorders $[2,3,6]$, and that anxiety trait, therefore, might mediate some of the elevated comorbidity between anxiety disorders and depression [7], we argue in favor of the etiological model represented in Figure $1 \mathrm{~b}$ and against the model based on a parallel causation as represented in Figure 1a. However, the model in Figure 1b implies a 'deterministic' link between high anxiety trait and the development of depression, which is at odds with emerging information emphasizing the modulatory role of stressful life events, which we reflect in the expanded model presented in Figure 2.

Although the discovery of risk factors (e.g. genetic, personality and stress) is essential to progress on the understanding of depression, it does not explain, by itself, what mechanisms mediate vulnerability to develop the disorder and its progression. Very active research in recent years has identified essential elements (e.g. neurocognitive patterns and neuroendocrine alterations) that, although representing important milestones in the field, only provide partial explanations for the understanding of how high anxiety trait predisposes to depression. Likewise, intensive work on the intricate interactions between emotion, stress and cognition has provided, in turn, important hints about the key role of such interactions for the development of depression. However, this information has so far remained rather fragmented and no unifying theory has as yet been offered.

Here, we propose a unifying model that integrates major progress on converging but independent research fields and proposes a trajectory whereby individuals presenting the vulnerability trait of high anxiety can eventually develop depression. We first go through the major neurocognitive and neuroendocrine hallmarks of depression to then examine the essential factors in high anxiety trait that promote the transition from the phenotypic predisposition provided by the personality trait to the dysfunctional alterations linked to depressive psychopathology. 
(a)

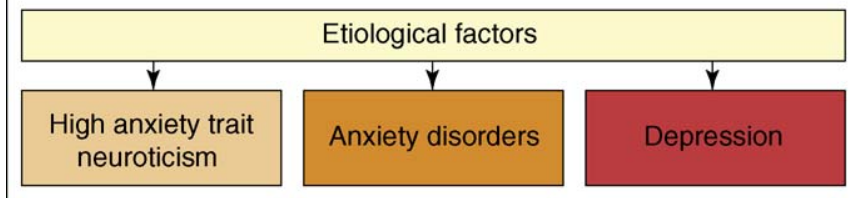

(b)



TRENDS in Neurosciences

Figure 1. Alternative etiological hypotheses for the development of depression. (a) According to this hypothesis, high anxiety trait, anxiety disorders and major depression are all directly caused by common etiological factors without any requirement for causal influences between the personality trait of anxiety and mood and anxiety disorders. (b) The second hypothesis proposes a first causal relationship between certain etiological factors and the development of the personality trait of high anxiety; in turn, this personality trait will be a vulnerable phenotype for the development of depression, either directly or via a first development of an anxiety disorder. Our model is based on this second etiological hypothesis.

Because exposure to stressful life events is a central element of the model, the latter is fed with essential information provided by research in the fields of emotion, stress and cognition, with a particular emphasis on an escalation of neuroendocrine and cognitive dysfunctions. The resulting neurocognitive model (Figure 3) enables the making of specific, testable predictions about which could be the most relevant therapeutic interventions at the different steps of the trajectory from high anxiety trait to depression.

\section{Neurocognitive and neuroendocrine hallmarks of depression}

Mood disturbances have been traditionally emphasized as core symptoms in depression, whereas cognitive alterations are considered to be simply epiphenomena (i.e. of age, poor motivation, inattention or response bias). However,

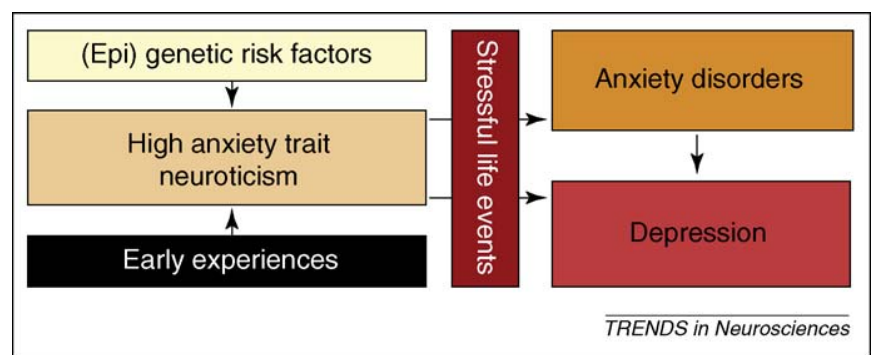

Figure 2. From high anxiety trait to depression: the etiological model. This figure depicts the factors that, according to our model, should converge for increased vulnerability to develop depression. Etiological factors [(epi)genetic and environmental (mainly early experiences)] first have a key role in defining the high anxiety trait phenotype. This personality trait confers a psychobiological vulnerability but does not necessarily cause, by itself, depression. The convergence of major stressful events will be the trigger (required factor in our model) that leads with a higher propensity in high anxious individuals than other personality types to develop depression, either directly or via a first development of an anxiety disorder. this traditional view has been challenged by recent suggestions that cognitive deficits are intrinsic expressions of the brain changes occurring in depressive illness [8] and by evidence showing that depression severity is correlated with the magnitude of cognitive deficits under some psychopathological conditions [9].

In fact, cognitive theories of depression have proposed now for some time [10] that negative cognitions are essential for the etiology and progression of the disorder. Cognitive disturbances in depressive patients are characterized by (i) enhanced negative cognitions (i.e. mood-congruent biases, which are related to a predisposition to form and particularly retrieve memories for negative information) and (ii) several cognitive deficits (in attention, executive function, working memory, and in both the short- and long-term memory formation) that might be instrumental for the expression of depression [8,11].

Another important feature in depression is a dysregulation of the hypothalamus-pituitary-adrenocortical (HPA) axis [12]. Many depressed subjects display enhanced cortisol secretion as a consequence of an overactive HPA axis [13], and enhanced activity of corticotropin-releasing-factor $(\mathrm{CRF})$ systems in limbic regions has been related with increased depression-like symptomatology [14].

\section{Neurocognitive characteristics of high anxiety trait}

Recent progress on the characterization of the neurocognitive profile corresponding to high anxiety trait reveals important neuropsychological features [15] that can partly explain why this trait represents a vulnerable phenotype to develop depression.

At the cognitive level, highly anxious individuals show a distinctive cognitive style characterized by alterations in the processing of threat, including bias in selective attention towards threat-related stimuli, interpretation of emotionally ambiguous stimuli as negative and enhanced fear conditioning responses [15]. Note the similarity between these cognitive alterations and a part of those characteristic of depressive patients, as mentioned in the previous section. In anxiety disorders, neuropsychological deficits have been reported at the level of executive function, attention, working memory and new learning [11].

At the level of functional neural dynamics, emphasis has concentrated on two types of abnormalities presented in high anxious subjects while processing emotional information: one related to the activation of the amygdala and the second related to amygdala coupling with other brain structures.

Neuroimaging studies initially observed a particular sensitivity of the amygdala to be activated when individuals from the general population are confronted with emotionally arousing material, with activation intensity correlating with the emotionality rate given to the material $[16,17]$. Other sets of studies also noted enhanced amygdala activation when individuals were exposed to unattended, masked or perceptually suppressed threat-related stimuli, implicating the amygdala in the rapid preattentive detection of threat $[15,18]$. In both research lines, studies relied on group analyses and, therefore, suggested that amygdala activation is a response usually shown 
when individuals are confronted with emotional or potentially threatening material. This work was subsequently complemented by evidence indicating that highly anxious individuals are particularly prone to respond to emotional stimulation with enhanced amygdala activation. This seems to hold true both when state and trait anxiety are considered, as shown by Bishop et al. [19] and Etkin et al. [20]. Bishop et al. [19] found that low anxious participants show a reduced amygdala response to unattended versus attended fearful faces, whereas high anxious participants show no such reduction, displaying an increased amygdala response to fearful versus neutral faces regardless of their attentional focus. Etkin et al. [20] showed that, indeed, individual differences in trait anxiety predicted both activity in the basolateral (but not dorsal) amygdala to unconscious stimuli and subjects' reaction times, with increased activity observed in individuals with higher anxiety trait score. Interestingly, a positive relationship between state anxiety and amygdala responses to neutral faces was also found in another study [21], which raises the possibility that high anxiety might bias the interpretation (as mediated by amygdala reactivity) of 'neutral' stimuli. Recent neuroimaging analyses in young rhesus monkeys provided further support for the existence of a link between high trait anxiety and increased amygdala reactivity, manifested not only under stressful conditions but also under more familiar ones [22]. These latter findings suggest that the alterations in amygdala responsiveness in highly anxious individuals are present from early life. Moreover, neuroimaging studies have also shown increased amygdala activity in response to negative emotional cues (e.g. fear, anger and sadness) in patients with anxiety disorders [23].

Together, these findings indicate a link between individual differences in trait anxiety and the proneness of the amygdala to respond to emotional stimuli and highlight the (basolateral) amygdala as a key area on the unconscious emotional vigilance characteristic of anxiety. They also suggest that high anxiety trait individuals respond as threatening to situations that others perceive as non stressful, which could reflect their lower threshold to interpret situations as adverse.

Imaging genomic studies have also shed light on the neurocognitive characteristics of the high anxiety trait. In 2002, a hallmark paper by Hariri et al. [24] reported a link between a functional polymorphism of the serotonin transporter (5-HTT, SLC6A4) gene and amygdala responses to fearful stimuli, with individuals presenting the short (S) allele of the gene exhibiting greater amygdala activity than individuals homozygous for the long (L) allele. These findings have been replicated and complemented by multiple subsequent studies [25-27]. They strongly support a genetic basis underlying differential excitability of the amygdala to emotional stimuli that might contribute to individual differences in emotional responses involving fear and anxiety. Interestingly, this genetic polymorphism was found to be associated with anxiety trait [28], with the $\mathrm{S}$ allele being associated with increased anxiety levels. Bertolino et al. [25] found that amygdala response during the presentation of threatening stimuli was predicted by anxiety-related personality style and as a function of S 5-
$H T T$ alleles. Very recently, another common genetic polymorphism affecting serotonin signaling [HTR1A $C(-1019) G]$ has also been found to predict interindividual variability in trait anxiety through its effects on amygdala reactivity but independently of the effects of the 5-HTT polymorphism [29]. Altogether, these studies might implicate increased serotonin signaling in driving amygdala reactivity and trait anxiety.

Although much attention has been given to the role and involvement of the amygdala in emotional responses and to its contribution to individual differences and to trait anxiety, it is clear that the amygdala exerts its effects through a network of dynamic interactions with other brain regions, mainly including the prefrontal cortex and the hippocampus. Human imaging studies clearly indicate that the balance activity within the amygdala-prefrontal circuitry is altered in anxiety and might contribute to the reported bias of high anxiety trait individuals to focus on and give greater weight to negative experiences [15]. Likewise, during perceptual processing of fearful stimuli, healthy carriers of the S 5-HTT allele presented abnormal uncoupling in the amygdala-cingulate feedback circuit, which has been implicated in the extinction of a negative affect [27]. Moreover, reduced ability of the prefrontal cortex to modulate amygdala reactivity has been associated with impaired extinction and a resultant hyperanxiety in both human and animal studies [30].

\section{Neuroendocrine reactivity in high anxiety trait}

Although there are inconsistencies in the literature with regards to the existence of a relationship between trait anxiety and stress responses, some reports have provided evidence for its existence. Rats psychogenetically selected for their high anxiety behavior showed stress-induced hyper-reactivity of the HPA axis, including increased glucocorticoid levels [31]. Healthy human subjects with high neuroticism displayed significantly greater levels of salivary cortisol in the post-waking period than those with lower neuroticism [32]. In adolescents, differences in cortisol levels were also found in the evening, with high anxious adolescents showing higher levels than low anxious adolescents [33]. During task performance, a moderator effect of trait anxiety on cortisol secretion was observed, suggesting that HPA activation due to performance pressure is stronger in subjects scoring high on anxiety measures, an effect that was understood to be potentially related to the increased attendance to threatening information and the interpretation of ambiguous events in a threatening way in high anxious individuals [34].

Moreover, emotionality and amygdala activation are associated with the activation of the HPA axis and with increased circulating glucocorticoids [35]. Conversely, the impact of enhanced amygdala activation might be further potentiated by a related increased activity of the HPA axis. Glucocorticoids increase the activity of neurons in the basolateral amygdala (BLA) $[36,37]$ and activate the production and release of $\mathrm{CRF}$ in the central nucleus $(\mathrm{CeA})$ of the amygdala and prefrontal cortex [38]. Infusion of corticosterone into the CeA increases anxiety in rats. Subjects with higher endogenous cortisol levels had significantly 
stronger amygdala responses to emotional slides when compared with subjects with lower cortisol levels [39].

\section{Memory modulation by emotionality}

Learning experiences triggering high emotionality and/or stress levels frequently lead to stronger memories than non stressful ones [17,40,41]. Emotional potentiation of memory was shown to involve and require [42] the activation of the amygdala during the processing of emotional stimuli. Even more, the level of activation of the amygdala was found to be positively correlated (both inter- and intrasubjects) with the degree at which the memory for those stimuli is later recovered [17]. Likewise, in rats, enhanced amygdala activation induced by high emotionality conditions was associated with faster acquisition and stronger memory for the task $[43,44]$.

The amygdala-enhancing effect on memory formation is believed to involve its interactions with other structures [45], particularly the hippocampus and prefrontal cortex $[46,47]$. The amygdala, activated by an emotionally charged event, would signal to other brain structures that the ongoing emotional information is 'worth' storing. This 'emotional tag' will strengthen synapses that have just been activated in another brain-memory system that is engaged in the learning situation eventually leading to the reinforcement of consolidation of that event [47,48]. In support of this view, recent work has shown that suppression of the amygdala to hippocampal effective connectivity blocks the emotion-induced memory-enhancing effect [49].

By contrast, enhanced memory for emotionally laden content has been shown to come together with a trade-off for non-emotionally laden information to be impaired when occurring just before the time of presentation of the emotional ones; that is, emotion-induced retrograde amnesia [50]. A potential contributing mechanism could be the differential enhancing or suppressing effects stress and amygdala activation have on neural plasticity in different hippocampal regions and in the amygdala itself [37,51,52].

Interestingly, recent work has shown that this phenomenon is specifically presented in individuals with the 5$H T T \mathrm{~S} / \mathrm{S}$ genotype [53]. In the same direction, in rats, highly anxious individuals were found to display impaired acquisition in a spatial learning task [54].

Glucocorticoids (stress hormones the release of which by the adrenal glands is increased by stress) have also been implicated in the potentiation of memory by stress and emotion $[40,41,55,56]$. Glucocorticoid administration can potentiate memory formation for mildly arousing experiences $[40,57]$ and the same glucocorticoid treatment was found to lead to increased reactivity of the basal amygdala [37], in a similar way as found in animals trained under highly arousing conditions [37,43]. However, it is important to emphasize that, whereas stress and glucocorticoids tend to exert positive effects on consolidation (especially for arousing material), they usually impair memory retrieval and working memory both in animals and humans $[57,58,59]$.

In summary, we propose that emotional learning will contribute to the development of psychopathology through mechanisms involving stress- and anxiety-induced impairment in hippocampus-prefrontal cortex processing, while enhancing of amygdaloid processing. Therefore, high emotionality would prime the storage of emotional material while interfering with the recall of previously acquired information and/or with the online manipulation of information (working memory).

In turn, sustained, chronic stress has been shown to impair hippocampal [60,61] and prefrontal cortex [62] structure and function while inducing the opposite, potentiating effects on the amygdala [63,64], thus bringing to extreme differential mechanisms that are found under brief moderate stress experiences [36,37,51,52]. These dissociated effects on different brain regions are paralleled by deficits in hippocampus-dependent learning $[65,66]$ and prefrontal-cortex-related working memory and behavioral flexibility [62], while facilitating amygdala-related processes such as fear conditioning [67]. We suggest that such structural and functional alterations in the hippocampus, prefrontal cortex and amygdala will be instrumental in the escalation of cognitive and emotional dysfunctions (and eventual development of affective disorders) triggered by exposure to stressful life events in highly anxious individuals.

\section{The impact of stress on the vulnerable phenotype of high anxiety trait}

While stressful life events are nowadays recognized to be key contributors for the development of depression [68], there is considerable variability in the susceptibility of individuals to the adverse effects of stress. We present evidence later to propose, in our model, that high anxiety trait individuals are a particularly prone phenotype in this context and that exposure to stressful life events is a requirement for individuals with this personality trait to develop depression (Figure 2).

Carriers of the S allele for the 5-HTT functional polymorphism-related to high neuroticism, when exposed to stressful life events of considerable magnitude, were found to display more severe depressive symptoms and to be at greater risk for a depressive episode than noncarriers (LL genotype); whereas S carriers not exposed to such stress levels did not develop depression [69,70] (see also Ref. [71]). Likewise, there are examples that such a gene-environment interaction also occurs in non-human primates. Work in female macaques indicates a particular susceptibility for carriers of the S allele that were, during early development, peer-only reared to develop high alcohol consumption, as compared with females from either the same genotype but reared by their mothers in social groups or with non-S allele carriers regardless of their rearing background [72]. In rhesus monkeys, a stressor context by genotype interaction was found such that the $\mathrm{S}$ allele influences different brain regions (including the amygdala) during exposure to different stressful situations [73]. In rodents, 5-HTT-deficient mice of at least one particular genetic background (C57BL/6J) only exhibit increased depression-related behavior after repeated exposure to uncontrollable stress [74].

Recent work in rodents also indicates a particular vulnerability of high anxious individuals to chronic stress exposure. The basolateral amygdala has a key role in emotional arousal and anxiety and contains high levels 
of CRF receptor-1 (CRFR1). In the rat, afferent activation of the BLA is increased after application of exogenous CRF [75]. This CRF-induced potentiation of afferent activation of the BLA was reduced in rats exposed to chronic unpredictable stress as compared with non-stressed controls. Interestingly, the reduction in BLA response to CRF was correlated with the anxiety trait of the animals determined before initiation of the chronic stress, with high anxious animals exposed to chronic stress showing the most disturbed amygdala responses to CRF [64]. These results suggest a vulnerability of high anxiety trait individuals to develop alterations in amygdaloid CRF systems under exposure to chronic stress. Moreover, high anxiety trait animals were the only ones that showed reduced levels of hippocampal cell proliferation after exposure to chronic stress [64], a phenomenon that has been suggested to be related to depression [76]. These results indicate a particular vulnerability of high anxiety trait animals to show stress-related alterations (non-stressed ones were not affected) in behavior and in functional responses in the amygdala and hippocampus.

\section{A neurocognitive model: from high anxiety trait to depression}

Evidence presented here prompted us to present a model that proposes neurocognitive styles associated with trait anxiety, and the neurobiological systems that sustain them, as the basis for the development of core symptoms of depression when (and mainly only 'when') individuals are confronted with stressful conditions. Gaining insight into the processes that underlie increased vulnerability to develop depression and the turning of that vulnerability onto a raising process of neurocognitive maladaptations should aid identifying potential therapeutic actions, not only for treatment of depression but, importantly, also for its prevention. Taking into consideration all lines of evidence described in the different sections before, we propose the following model to account for the predisposition of high anxiety individuals to develop depression (Figure 3).

(i) High anxiety trait individuals show an attentional bias to detect aversiveness that is accompanied by increased amygdala reactivity under both pre-attentive and selective attention conditions. In consequence, highly anxious individuals tend to perceive even moderate events as stressful and their neuropsychological responses to threat or negative stimuli are stronger than low anxious individuals.

(ii) This hyper-responsiveness to threat (both in terms of behavioral responses and amygdala activation) make highly anxious individuals particularly prone to experience fear and stress, with the consequent activation of the HPA axis and increased glucocorticoid levels.

(iii) Enhanced amygdala activation and HPA axis reactivity contribute to enhanced emotional memory consolidation, leading in the case of highly anxious individuals to increased storage of both fear associations (through classical conditioning) and of negative emotional episodic memories. This characteristic pattern of responses and processes defines a vulnerability phenotype of which the evolution towards depression does not usually occur without further triggering factors.

(iv) When confronted with major life stressors, the vulnerable neurocognitive phenotype of highly anxious individuals acts as a sensitized system that displays an exaggerated attentional focus to the 'negative' side of events and has all the elements to form strong aversive memories.

(v) When facing stress, this turns the negativity-bias 'internal milieu' of highly anxious individuals into a 'mood-congruent bias', which refers to their increased propensity to have negative thoughts, to primarily attend to negative circumstances and to tend to primarily remember negative episodic memories or events.

(vi) In parallel, amygdala and glucocorticoid hyperactivity linked to such negative processing will increasingly impair certain hippocampus- and prefrontal-cortex-related functions, leading to a series of cognitive deficits on attention, concentration, working memory, new learning and executive function.

(vii) Both the reverberant negativity bias and the cognitive deficits will render stressed, highly anxious individuals ineffective to cognitively (and actually) cope with everyday life challenges. This will quickly reinforce the feelings of hopelessness, helplessness and worthlessness (characteristics of depression) in these individuals. Myriad physiological alterations typical of depression (e.g. altered patterns of appetite, sleep and body weight) will follow.

(viii) All these dysfunctional changes experienced by highly anxious individuals under stressful conditions will markedly affect their motivation in many life domains and the consequent behavioral withdrawal. These alterations will result in a loss of reinforcements (social, others) that will strongly exacerbate depressive mood. In turn, depressive mood will act on the same neurobiological and neuroendocrine substrates that participated in the development of depression. This will result on a loop that chronifies the depression episode into a so called depression disorder.

This model leads to several predictions with important implications for the development of therapeutic interventions (prevention and or treatment) that could be applied at the different steps of the trajectory from high anxiety trait to depression. For example, development of treatments capable of restraining amygdala activation (either directly or, for example, by enhancing inhibitory activity from the prefrontal cortex) warrants successful effects at several phases of the trajectory. However, a note of caution should be drawn about the generality of such treatments. It might be that an approach that is effective to upraise the threshold for amygdala activation to neutral-to-aversive or fearful stimuli (as occurring in healthy subjects with high anxiety trait) is not sufficient to 'cool down' an amygdala that is tonically hyper-reactive (as shown for depressive individuals). This might imply the need to explore alternative approaches for each of these conditions. 


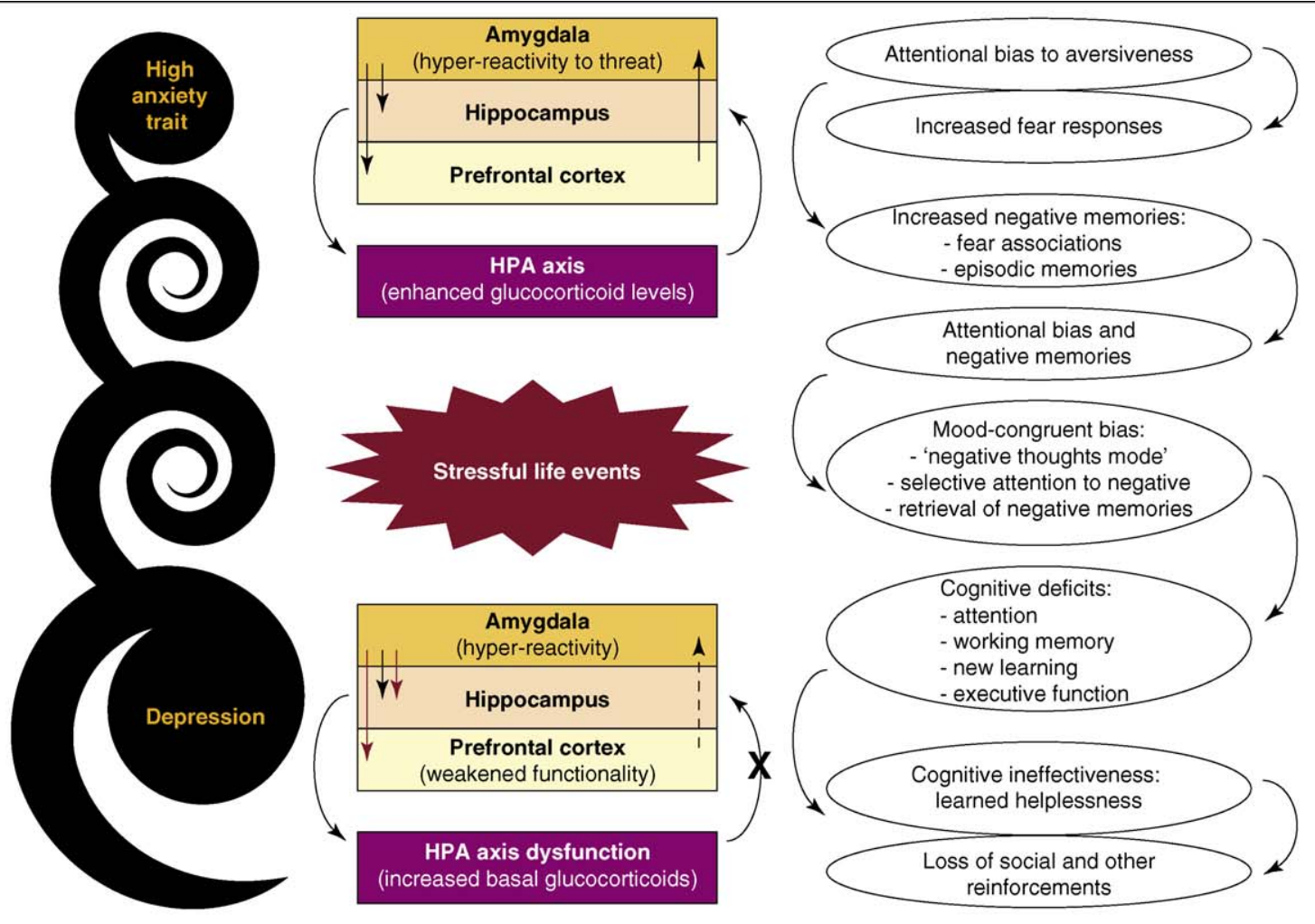

TRENDS in Neurosciences

Figure 3. From high anxiety trait to depression: a neurocognitive model. High anxiety trait individuals display enhanced amygdala reactivity that will influence activity in the hippocampus and prefrontal cortex (black arrows); conversely, the prefrontal cortex exerts a weak inhibition of amygdala function. This profile is linked with a phasic enhancement of hypothalamus-pituitary-adrenal (HPA) axis activity and consequently of glucocorticoid levels. Conversely, phasic elevations of glucocorticoids further increase amygdala activation. This pattern of brain and neuroendocrine interactions (middle column) is accompanied, in high anxiety trait individuals, by a cognitive pattern of interrelated responses (right column). When high anxious individuals are exposed to major stressful life events, this vulnerable neurocognitive profile responds with amygdala sensitization (hyper-reactivity to threat that progressively chronifies to hyperactivation even under basal conditions) with stronger influence over hippocampal and prefrontal (strongly potentiating [black arrows] or inhibiting [red arrows]) functions. The prefrontal cortex becomes ineffective to restrain amygdala hyper-reactivity (dotted arrow). This pattern of neural dynamics disrupts functionality of the HPA axis, which eventually shows enhanced glucocorticoid levels under basal conditions. Conversely, high glucocorticoids further disrupt hippocampal and prefrontal cortex function. In turn, this neural and neuroendocrine pattern (middle column) evolved as a consequence of sustained stress exposure turns the cognitive style into a spiral of progressively enhanced dysfunctions (right columns). The focus on threat and fear results in enhanced negative memories, setting the cognitive processing in a 'mood-congruent' bias mode that together with cognitive deficits of increasing severity leads to several socio-psycho-physiological alterations that are at the code of depression. The neurocognitive pattern characteristic of high anxious individuals determines the increasing and reverberating nature of these maladaptive neurocognitive processes and sets the path to turn a depression episode into a major depression disorder.

The model also leads to the hypothesis that approaches addressed to inhibit the degree of glucocorticoid activation might help to impede the escalation of cognitive dysfunctions. Along with pharmacological treatments that inhibit glucocorticoid synthesis and release or that antagonize glucocorticoid receptor function, any other procedure (e.g. psychotherapy, relaxation, exercise, nutrition, etc) capable of affecting glucocorticoid function might also be effective.

This model also implies that cognitive training addressed to reinforce hippocampal and prefrontal-cortex-related functions might be effective in containing the development of depression in highly anxious individuals. Finally, our model particularly emphasizes the importance of developing and testing treatments addressed to target the highlighted memory dysfunctions (i.e. to interfere with the quality and/or intensity of consolidation and retrieval of strongly aversive emotional memories; to contain the strength of fear conditioning processes and/or promote their extinction; to exercise declarative and working memory functions).

\section{Concluding remarks}

Support for this model has been recently provided by neuroimaging studies indicating that motivation and stress-related systems that include the amygdala are pathologically activated in major depression [77] and that this activity is associated with (i) an automatic judgmental bias towards negativity [78]; (ii) altered emotion processing [79]; (iii) greater memory sensitivity than non-depressed participants to negative but not to neutral or positive stimuli, with the degree of memory-related amygdala activation in the depressed patients being correlated with depression severity [80]; and (iv) dysfunction of the prefrontal cortex and monoamine neurotransmitter systems that normally modulate such responses [81]. Enhanced activity in the amygdala was found to be positively correlated with plasma cortisol levels in patients with depression [82]. Depressed patients carrying the S 5-HTT gene alleles also show increased amygdala activity compared with depressed non-risk allele carriers, even when emotional stimuli are masked and not accessible to conscious awareness [78]. Likewise, depression is associated 
with prefrontal-cortex-related cognitive deficits, particularly of working memory [8]. These are not independent outcomes. Increasing evidence suggests that the ability of the medial prefrontal cortex to modulate anxiety-related output from the amygdala is compromised in depressed people [83].

However, it should be noted that a more specific and detailed analysis of the exact nature of amygdala activation is required before its role and nature of interaction with the prefrontal cortex under normal and pathological conditions is delineated. Higher rather than lower event-related amygdala amplitude was found to predict faster response time, with no loss of accuracy in a working memory task; this is a relationship that was not contingent on mood state, task content or personality variables [84]. Furthermore, increased activation in the amygdala has recently been linked to the detection of behaviorally relevant stimuli in normal human subjects [85].

This novel unifying model highlights the importance of the convergence of the vulnerability phenotype of high anxiety trait and exposure to stressful life events for the development of depression. It identifies a maladaptive neurocognitive system that predisposes to multiply the impact of adversity on these individuals. It therefore points out the importance of developing prevention programs (e.g. cognitive-emotional therapy, stress-reduction approaches and biofeedback potentially coupled to neuroimaging and psychophysiological approaches) to help high anxious individuals cope with stress and to prevent the triggering of the dysfunctional neurocognitive cascade. In consequence, this model advocates the early identification of high anxious individuals, and certainly before they are exposed to major stressful events, to effectively fight the burden of depression. As a much larger proportion of the population falls into the category of high anxiety than those that eventually develop clinical depression, future efforts to optimize identification of individuals at risk to develop depression will need to combine other indexes or markers (i.e. other personality traits; genetic, biological and environmental factors). According to our model, particular biases in neuroendocrine reactivity and memory characteristics would synergize with high anxiety trait profiles in precipitating depressive dysfunction. Genetic, neuroendocrine and neuropsychological studies will, therefore, need to be combined to improve recognition of individuals at risk. In the meantime, our model encourages therapeutic programs to pay particular attention to the tackling of memory distortions (particularly potentiation of aversive memories, fear conditioning and impaired extinction, working memory alterations and state-dependent retrieval of aversive memories) developed by highly anxious individuals when exposed to different sources of stress.

\section{Acknowledgements}

This work has been partially supported by grants from the Swiss National Science Foundation (to C.S.; www.snf.ch), FP6 (PROMEMORIA; to C.S. and G.R.L.; http://plab.ku.dk/promemoria) and FP7 (MemStick; to C.S.; www.memstick.org) EU programmes, and a research grant from the Institute for the Study of Affective Neuroscience (to G.R.L.; http:// isan.hevra.haifa.ac.il).

\section{References}

1 Duggan, C. et al. (2003) Theories of general personality and mental disorder. Br. J. Psychiatry Suppl. 44 (Suppl.), S19-S23

2 Kendler, K.S. et al. (2004) The interrelationship of neuroticism, sex, and stressful life events in the prediction of episodes of major depression. Am. J. Psychiatry 161, 631-636

3 Hettema, J.M. (2008) What is the genetic relationship between anxiety and depression? Am. J. Med. Genet. C. Semin. Med. Genet. 148C, 140-146

4 Kessler, R.C. et al. (2003) The epidemiology of major depressive disorder: results from the National Comorbidity Survey Replication (NCS-R). J. Am. Med. Assoc. 289, 3095-3105

5 Bittner, A. et al. (2004) What characteristics of primary anxiety disorders predict subsequent major depressive disorder? J. Clin. Psychiatry 65, 618-626

6 Merikangas, K.R. and Pine, D. (2002) Genetic and other vulnerability factors for anxiety and stress disorders. In Neuropharmacology: The Fifth Generation of Progress (Davis, K.L. and et, al., eds), pp. 867-882, American College of Neuropsychopharmacology

7 Leonardo, E.D. and Hen, R. (2006) Genetics of affective and anxiety disorders. Annu. Rev. Psychol. 57, 117-137

8 Austin, M.P. et al. (2001) Cognitive deficits in depression. Br. J. Psychiatry 178, 200-206

9 Hill, S.K. et al. (2004) Neuropsychological dysfunction in antipsychoticnaive first-episode unipolar psychotic depression. Am. J. Psychiatry 161, 996-1003

10 Beck, A.T. (1976) Cognitive Therapy and the Emotional Disorders. International Universities Press

11 Castaneda, A.E. et al. (2008) A review on cognitive impairments in depressive and anxiety disorders with a focus on young adults. J. Affect. Disord. 106, 1-27

12 Foster, J.A. and MacQueen, G. (2008) Neurobiological factors linking personality traits and major depression. Can. J. Psychiatry 53, 6-13

13 Pariante, C.M. and Lightman, S.L. (2008) The HPA axis in major depression: classical theories and new developments. Trends Neurosci. 31, 464-468

14 Reul, J.M. and Holsboer, F. (2002) Corticotropin-releasing factor receptors 1 and 2 in anxiety and depression. Curr. Opin. Pharmacol. $2,23-33$

15 Bishop, S.J. (2007) Neurocognitive mechanisms of anxiety: an integrative account. Trends Cogn. Sci. 11, 307-316

16 Cahill, L. et al. (1994) $\beta$-adrenergic activation and memory for emotional events. Nature 371, 702-704

17 Canli, T. et al. (2000) Event-related activation in the human amygdala associates with later memory for individual emotional experience. $J$. Neurosci. 20, RC99

18 Dolan, R.J. and Vuilleumier, P. (2003) Amygdala automaticity in emotional processing. Ann. N. Y. Acad. Sci. 985, 348-355

19 Bishop, S.J. et al. (2004) State anxiety modulation of the amygdala response to unattended threat-related stimuli. J. Neurosci. 24, 1036410368

20 Etkin, A. et al. (2004) Individual differences in trait anxiety predict the response of the basolateral amygdala to unconsciously processed fearful faces. Neuron 44, 1043-1055

21 Somerville, L.H. et al. (2004) Human amygdala responses during presentation of happy and neutral faces: correlations with state anxiety. Biol. Psychiatry 55, 897-903

22 Fox, A.S. et al. (2008) Trait-like brain activity during adolescence predicts anxious temperament in primates. PLoS One 3, e2570

23 McClure, E.B. et al. (2007) Abnormal attention modulation of fear circuit function in pediatric generalized anxiety disorder. Arch. Gen. Psychiatry 64, 97-106

24 Hariri, A.R. et al. (2002) Serotonin transporter genetic variation and the response of the human amygdala. Science 297, 400-403

25 Bertolino, A. et al. (2005) Variation of human amygdala response during threatening stimuli as a function of 5-HTTLPR genotype and personality style. Biol. Psychiatry 57, 1517-1525

26 Heinz, A. et al. (2005) Amygdala-prefrontal coupling depends on a genetic variation of the serotonin transporter. Nat. Neurosci. 8, 20-21

27 Pezawas, L. et al. (2005) 5-HTTLPR polymorphism impacts human cingulated-amygdala interactions: a genetic susceptibility mechanism for depression. Nat. Neurosci. 8, 828-834

28 Schinka, J.A. et al. (2004) A meta-analysis of the association between the serotonin transporter gene polymorphism (5-HTTLPR) and trait anxiety. Mol. Psychiatry 9, 197-202 
29 Fakra, E. et al. (2009) Effects of HTR1A C(-1019)G on amygdala reactivity and trait anxiety. Arch. Gen. Psychiatry 66, 33-40

30 Hofmann, S.G. (2008) Cognitive processes during fear acquisition and extinction in animals and humans: implications for exposure therapy of anxiety disorders. Clin. Psychol. Rev. 28, 199-210

31 Landgraf, R. and Wigger, A. (2003) Born to be anxious: neuroendocrine and genetic correlates of trait anxiety in HAB rats. Stress 6, 111-119

32 Portella, M.J. et al. (2005) Enhanced early morning salivary cortisol in neuroticism. Am. J. Psychiatry 162, 807-809

33 Van den Bergh, B.R. et al. (2008) Self-reported symptoms of depressed mood, trait anxiety and aggressive behavior in post-pubertal adolescents: associations with diurnal profiles. Horm. Behav. 54, 253-257

34 Schlotz, W. et al. (2006) Trait anxiety moderates the impact of performance pressure on salivary cortisol in everyday life. Psychoneuroendocrinology 31, 459-472

35 Rohleder, N. et al. (2003) Glucocorticoid sensitivity in humansinterindividual differences and acute stress effects. Stress 6, 207-222

36 Duvarci, S. and Pare, D. (2007) Glucocorticoids enhance the excitability of principal basolateral amygdala neurons. J. Neurosci. 27, 4482-4491

37 Kavushansky, A. and Richter-Levin, G. (2006) Effects of stress and corticosterone on activity and plasticity in the amygdala. J. Neurosci. Res. 84, 1580-1587

38 Merali, Z. et al. (2008) Effects of corticosterone on corticotrophinreleasing hormone and gastrin-releasing peptide release in response to an aversive stimulus in two regions of the forebrain (central nucleus of the amygdala and prefrontal cortex). Eur. J. Neurosci. 28, 165-172

39 Van Stegeren, A.H. et al. (2006) Endogenous cortisol levels interacts with noradrenergic activation in the human amygdala. Neurobiol. Learn. Mem. 87, 57-66

40 Sandi, C. et al. (1997) Experience-dependent facilitating effect of corticosterone on spatial memory formation in the water maze. Eur. J. Neurosci. 9, 637-642

41 Cordero, M.I. et al. (1998) Correlational relationship between shock intensity and corticosterone secretion on the establishment and subsequent expression of contextual fear conditioning. Behav. Neurosci. 112, 885-891

42 Adophs, R. et al. (1997) Impaired declarative memory for emotional material following bilateral amygdala damage in humans. Learn. Mem. 4, 291-300

43 Akirav, I. et al. (2001) Differential activation of hippocampus and amygdala following spatial learning under stress. Eur. J. Neurosci. $14,719-725$

44 Kogan, I. and Richter-Levin, G. (2008) Activation pattern of the limbic system following spatial learning under stress. Eur. J. Neurosci. 27, $715-722$

45 McGaugh, J.L. (2002) Memory consolidation and the amygdala: a systems perspective. Trends Neurosci. 25, 456

46 Richardson, M.P. et al. (2004) Encoding of emotional memories depends on amygdala and hippocampus and their interactions. Nat. Neurosci. 7, 278-285

47 Richter-Levin, G. (2004) The amygdala, the hippocampus, and emotional modulation of memory. Neuroscientist 10, 31-39

48 Richter-Levin, G. and Akirav, I. (2003) Emotional tagging of memory formation - in the search for neural mechanisms. Brain Res. Brain Res. Rev. 43, 247-256

49 Alkire, M.T. et al. (2008) Neuroimaging analysis of an anesthetic gas that blocks human emotional memory. Proc. Natl. Acad. Sci. U. S. A. $105,1722-1727$

50 Strange, B.A. et al. (2003) An emotion-induced retrograde amnesia in humans is amygdala- and $\beta$-adrenergic-dependent. Proc. Natl. Acad. Sci. U. S. A. 100, 13626-13631

51 Vouimba, R.M. and Richter-Levin, G. (2005) Physiological dissociation in hippocampal subregions in response to amygdala stimulation. Cereb. Cortex 15, 1815-1821

52 Kavushansky, A. et al. (2006) Activity and plasticity in the CA1, the dentate gyrus, and the amygdala following controllable vs. uncontrollable water stress. Hippocampus 16, 35-42

53 Strange, B.A. et al. (2008) Emotion-induced retrograde amnesia is determined by a 5-HTT genetic polymorphism. J. Neurosci. 28, 7036-7039

54 Herrero, A.I. et al. (2006) Individual differences in anxiety trait are related to spatial learning abilities and hippocampal expression of mineralocorticoid receptors. Neurobiol. Learn. Mem. 86, 150-159
55 Roozendaal, B. et al. (2006) Glucocorticoids interact with emotioninduced noradrenergic activation in influencing different memory functions. Neuroscience 138, 901-910

56 de Kloet, E.R. et al. (1999) Stress and cognition: are corticosteroids good or bad guys? Trends Neurosci. 22, 422-426

57 Akirav, I. et al. (2004) A facilitative role for corticosterone in the acquisition of a spatial task under moderate stress. Learn. Mem. 11, 188-195

58 Kim, J.J. and Diamond, D.M. (2002) The stressed hippocampus, synaptic plasticity and lost memories. Nat. Rev. Neurosci. 3, 453-462

59 Luethi, M. et al. (2009) Stress effects on working memory, explicit memory, and implicit memory for neutral and emotional stimuli in healthy men. Front. Behav. Neurosci. 2, 5 DOI:0.3389/ neuro.08.005.2008 (www.frontiersin.org)

60 Magariños, A.M. et al. (1997) Chronic stress alters synaptic terminal structure in hippocampus. Proc. Natl. Acad. Sci. U. S. A. 94, 1400214008

61 Pavlides, C. et al. (2002) Effects of chronic stress on hippocampal longterm potentiation. Hippocampus 12, 245-257

62 Cerqueira, J.J. et al. (2007) The prefrontal cortex as a key target of the maladaptive response to stress. J. Neurosci. 27, 2781-2787

63 Vyas, A. et al. (2002) Chronic stress induces contrasting patterns of dendritic remodeling in hippocampal and amygdaloid neurons. $J$. Neurosci. 22, 6810-6818

64 Sandi, C. et al. (2008) Chronic stress-induced alterations in amygdala responsiveness and behavior-modulation by trait anxiety and corticotropin-releasing factor systems. Eur. J. Neurosci. 28, 1836-1848

65 Touyarot, K. et al. (2004) Spatial learning impairment induced by chronic stress is related to individual differences in novelty reactivity: search for neurobiological correlates. Psychoneuroendocrinology 29, 290-305

66 Sandi, C. (2004) Stress, cognitive impairment and cell adhesion molecules. Nat. Rev. Neurosci. 5, 917-930

67 Sandi, C. et al. (2001) Effects of chronic stress on contextual fear conditioning and the hippocampal expression of the neural cell adhesion molecule, its polysialylation, and L1. Neuroscience 102, 329-339

68 Kendler, K.S. et al. (1999) Causal relationship between stressful life events and the onset of major depression. Am. J. Psychiatry 156, 837841

69 Caspi, A. et al. (2003) Influence of life stress on depression: moderation by a polymorphism in the 5-TT gene. Science 301, 386-389

70 Kendler, K.S. et al. (2005) The interaction of stressful life events and a serotonin transporter polymorphism in the prediction of episodes of major depression: a replication. Arch. Gen. Psychiatry 62, 529-535

71 Surtees, P.G. et al. (2006) Social adversity, the serotonin transporter (5H-TTLPR) polymorphism and major depressive disorder. Biol. Psychiatry 59, 224-229

72 Barr, C.S. et al. (2004) Interaction between serotonin transporter gene variation and rearing condition in alcohol preference and consumption in female primates. Arch. Gen. Psychiatry 61, 1146-1152

73 Kalin, N.H. et al. (2008) The serotonin transporter genotype is associated with intermediate brain phenotypes that depend on the context of eliciting stressor. Mol. Psychiatry 13, 1021-1027

74 Zhao, S. et al. (2006) Insertion mutation at the C-terminus of the serotonin transporter disrupts brain serotonin function and emotion-related behaviors in mice. Neuroscience 140, 321-334

75 Ugolini, A. et al. (2008) CRF1 receptor activation increases the response of neurons in the basolateral nucleus of the amygdala to afferent stimulation. Front Behav. Neurosci. 2, 2

76 Duman, R.S. (2004) Depression: a case of neuronal life and death? Biol. Psychiatry 56, 140-145

77 Siegle, G.J. et al. (2007) Increased amygdala and decreased dorsolateral prefrontal BOLD responses in unipolar depression. Related and independent features. Biol. Psychiatry 61, 198-209

78 Dannlowski, U. et al. (2008) Amygdala reactivity predicts automatic negative evaluations for facial emotions. Psychiatry Res. 154, 13-20

79 Whalen, P.J. et al. (2002) Functional neuroimaging studies of the amygdala in depression. Semin. Clin. Neuropsychiatry 7, 234-242

80 Hamilton, J.P. and Gotlib, I.H. (2008) Neural substrates of increased memory sensitivity for negative stimuli in major depression. Biol. Psychiatry 63, 1155-1162

81 Drevets, W.C. (2003) Neuroimaging abnormalities in the amygdala in mood disorders. Ann. N. Y. Acad. Sci. 985, 420-444 
82 Drevets, W.C. et al. (2002) Glucose metabolism in the amygdala in depression: relationship to diagnostic subtype and stressed plasma cortisol levels. Pharmacol. Biochem. Behav. 71, 431447

83 Johnstone, T. et al. (2007) Failure to regulate: counterproductive recruitment of top-down prefrontal-subcortical circuitry in major depression. J. Neurosci. 27, 8877-8884
84 Schaefer, A. et al. (2006) Individual differences in amygdala activity predict response speed during working memory. J. Neurosci. 26, $10120-10128$

85 Ousdal, O.T. et al. (2008) The human amygdala is involved in general behavioral relevance detection: evidence from an event-related functional magnetic resonance imaging Go-NoGo task. Neuroscience $156,450-455$ 